\section{Quand trop de myélinisation détruit la myéline}

Sylvain Bartolami ${ }^{1,2}$, Nicolas Tricaud ${ }^{1}$
1 Équipe Avenir «myéline » Inserm U1051, institut des neurosciences de Montpellier, hôpital Saint-દ́loi, 80 , rue Augustin Fliche, 34091 Montpellier cedex 5, France.

${ }^{2}$ Université Montpellier 2, place Eugène Bataillon, 34095 Montpellier cedex 5, France. sylvain.bartolami@univ-montp2.fr
> L'augmentation de la vitesse de conduction nerveuse, grâce au processus de myélinisation, est un élément essentiel de l'émergence des vertébrés. Dans le système nerveux périphérique, les cellules de Schwann s'enroulent en plusieurs tours de spires membranaires compactes autour de l'axone qui constituent des manchons électriquement isolants - les gaines de myéline - séparés par les nœuds de Ranvier. La succession de ces gaines augmente la vitesse de conduction nerveuse car les potentiels d'action doivent sauter d'un nœud de Ranvier à un autre, ce qui accélère leur vitesse de propagation. L'épaisseur et la longueur de la gaine de myéline sont donc des paramètres essentiels de la conduction de l'influx nerveux. L'importance fonctionnelle de la myéline est particulièrement mise en exergue lorsque survient une réduction de la conduction nerveuse comme dans les pathologies démyélinisantes très handicapantes, par exemple les maladies de Charcot-Marie-Tooth (CMT) [1].

\section{Régulation de la myélinisation}

La myélinisation est propre aux axones de gros calibre (supérieur à $1 \mu \mathrm{m}$ ). L'étape de myélinisation est induite via la synthèse de facteurs trophiques dont le plus connu est la neuréguline 1 (NRGl) de type III [2]. Cette protéine transmembranaire est présentée par l'axone et interagit avec les récepteurs erythroblastic leukemia viral oncogene homolog 2/3 (erbB2/3) des cellules de Schwann [3, 4]. L'activation de ces récepteurs provoque la différenciation des cellules de Schwann immatures en cellules de Schwann myélinisantes, puis stimule la production de myéline par ces cellules [2] via la voie de signalisation de la phosphoinositide-3 kinase (PI3K) et de la protéine kinase $B$ (AKT) [5].

Certaines maladies de Charcot Marie Tooth se traduisent par un dépôt anormal de myéline sous forme d'accumulations focales qui suggèrent un excès de myélinisation. C'est notamment le cas lors de certaines mutations du gène codant pour la myotubularin-related protein 2 (MTMR2) dans la CMT de type 4B. Dans les nerfs de souris, MTMR2 interagit avec la protéine de polarité cellulaire Disks large homolog 1 (Dlgl), suggérant l'intervention d'un processus de polarisation de la cellule de Schwann lors de la myélinisation. Après avoir étudié la polarisation cellulaire des cellules de Schwann myélinisantes [6], nous avons montré que Dlgl et MTMR2 participaient à un mécanisme prévenant un excès de

\section{A ARNsh DIg1 + GFP}



B ARNsh cont + GFP
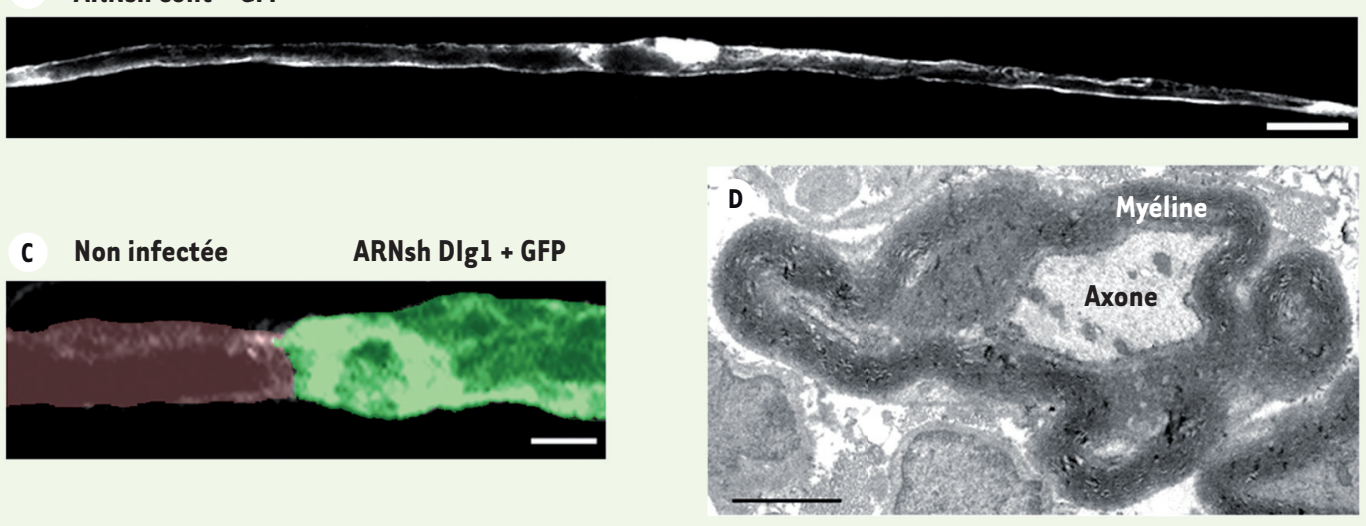

Figure 1. L'absence de Dlg1 in vivo provoque un excès de myélinisation. L'inhibition de l'expression de DIgl dans une cellule de Schwann infectée par des vecteurs exprimant un ARNsh dirigé contre DIgl (A. ARNsh Dlgl + GFP) entraîne un épaississement de la myéline par rapport à une cellule de Schwann infectée par des virus contrôles (B. ARNsh cont + GFP). C. Cet épaississement est particulièrement évident au niveau d'un nœud de Ranvier. $D$. Dans certaines fibres, l'inhibition de l'expression de Dlgl conduit à une myélinisation excessive aberrante (barres d'échelle en $A$ et $B: 20 \mu \mathrm{m}$; en $C: 5 \mu \mathrm{m}$; en $D: 2 \mu \mathrm{m}$ ) (d'après [7]). 
myélinisation dans ces cellules. Ces résultats ont été obtenus via une approche d'ARN interférence qui permet d'inhiber la synthèse d'une protéine cible à l'aide de short hairpin RNA (ARNsh) ciblant l'ARNm sur le RNA-induced silencing complex (RISC) [7]. Cette inhibition de l'expression d'une protéine est possible in vivo en utilisant des vecteurs viraux exprimant les ARNsh spécifiquement dans les cellules de Schwann [6]. De tels virus, injectés dans le nerf sciatique de souris nouveau-nés, ont été utilisés pour éteindre l'expression de Dlgl pendant la myélinisation. Privées de DIgl, les cellules de Schwann produisent des gaines de myéline significativement plus épaisses que celles que produisent des cellules de Schwann infectées avec un virus contrôle (Figure IA-C). À l'inverse, lorsque Dlgl est surexprimée dans les cel-
Iules de Schwann via un vecteur viral, celles-ci sont incapables de synthétiser la gaine de myéline. Ces résultats montrent que DIgl participe à un mécanisme d'inhibition de la myélinisation. Cependant, quel est son mode d'action?

\section{Le couple DIg1/PTEN :}

un frein à la myélinisation au cours du développement et chez l'adulte Nous nous sommes basés sur le fait que DIgl peut interagir avec la phosphatase with sequence homology to tensin (PTEN) qui inhibe l'activation d'AKT [8, 9]. Des expériences de co-immunoprécipitation ont confirmé que DIgl et PTEN interagissaient bien dans les cellules de Schwann [7]. De plus, la quantité de PTEN présente dans ces cellules était directement liée au niveau d'expression de Dlgl.
Ce résultat suggérait que DIgl pouvait contrôler la quantité de PTEN disponible, et donc l'activation d'AKT et la régulation de la myélinisation. En effet, l'inhibition in vivo de l'expression de PTEN induit un excès de myélinisation comparable à celui qui est observé lorsque DIgl est éteint, suggérant que ces deux facteurs coopèrent pour inhiber la myélinisation. L'utilité d'un tel mécanisme de régulation est manifeste lors du développement du nerf sciatique de la souris. En effet, douze jours après la naissance, l'épaisseur de la myéline commence à tendre vers l'optimal, et la quantité de DIgl et de PTEN atteint un pic qui s'accompagne ensuite d'un fléchissement du degré de myélinisation. Ce mécanisme de limitation de la myélinisation semble persister chez l'adulte car l'inhibition de l'expression de DIgl chez des souris plus
A Début de la myélinisation



\section{B Myélinisation optimale}



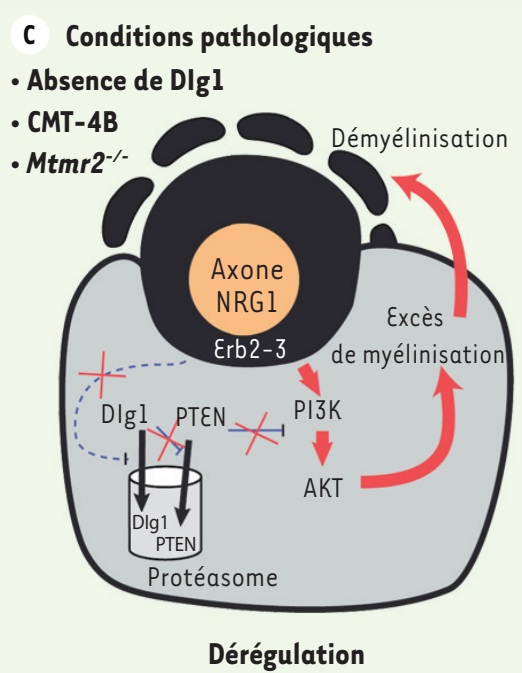

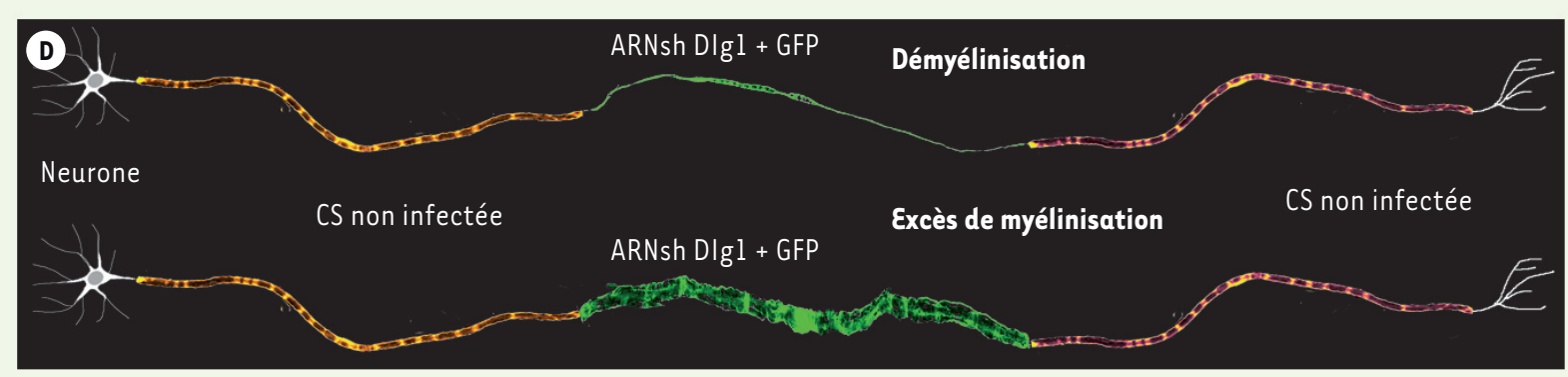

Figure 2. Contrôle différentiel de la myélinisation par la NRG1. A. Au début de la myélinisation, l'effet de la NRGl s'exprime essentiellement par I'activation de la voie promyélinisante PI3K/AKT. B. Lorsque le diamètre de la fibre atteint son optimum, l'effet inhibiteur de NRGl sur la dégradation de DIgl et PTEN permet l'accumulation de DIgl et PTEN. PTEN inhibe alors fortement l'activation d'AKT et freine la myélinisation. C-D. La perturbation de ce frein chez la souris (absence de Dlgl et mutant Mtmr2 ${ }^{-/-}$) et chez les patients atteints de la maladie de CMT de type 4B (CMT-4B) entraîne un excès de myélinisation suivie d'une démyélinisation. CS : cellule de Schwann. 
âgées induit également la production de manchons de myéline hypertrophiés (Figure 1D) qui finissent par dégénérer [7]. Le freinage exercé par Dlgl sur la myélinisation est donc nécessaire pour éviter un excès de myélinisation puis une démyélinisation. Mais qui enclenche ce frein?

\section{Défaut du frein de la myélinisation exercé par Dlg1 dans les CMT 4B}

Compte tenu de son rôle clé dans la myélinisation, l'influence de NRGl sur le duo DIgl/PTEN a été étudiée. De fait, les quantités de DIgl et de PTEN s'accroissent lorsque les cellules de Schwann sont stimulées par la NRGl. En effet, cette dernière inhibe l'ubiquitinylation de DIgl et de PTEN, bloquant leur dégradation par le protéasome. En conséquence, les deux protéines ainsi protégées s'accumulent et exercent une inhibition croissante sur la voie PI3K/AKT. Lorsque DIgl devient prédominant - ce qui arrive vers la fin de la $3^{e}$ semaine de vie chez la souris -, la myélinisation est contrainte de s'arrêter. Ainsi la NRGl active deux voies antagonistes: I'une, via PI3K/AKT, stimule la myélinisation ; l'autre, via DIgl et PTEN, la stoppe (Figure 2A-B).

Mais notre résultat le plus marquant [7] est d'avoir établi un lien entre cette régulation de la myélinisation et certaines CMT. En effet nous avons effacé DIgl dans le nerf sciatique de souris dont le gène Mtmr2 a été invalidé et qui représentent donc un modèle de la CMT de type 4B [10]. La suppression de DIgl n'induit pas d'excès de myélinisation indiquant que, chez ces mutants CMT$4 B$, DIgl n'exerce pas son rôle de frein de la myélinisation. Ces résultats suggèrent que la défaillance du mécanisme inhibant la myélinisation est à l'origine de l'excès de myélinisation qui s'accompagne d'une démyélinisation caractéristique la CMT de type 4B (Figure 2C-D).

En résumé, au cours du développement, une myélinisation optimale des axones nécessite un processus de freinage, reposant sur DIgl et PTEN, couplé, via NRGl, au processus de stimulation axonale. Dans les nerfs matures, la perte de fonction de Digl provoque un excès de myélinisation suivi d'une démyélinisation. La perturbation de ce mécanisme de freinage pourrait donc jouer un rôle majeur dans la physiopathologie des neuropathies démyélinisantes caractérisées par une myélinisation excessive focale comme on l'observe dans la maladie de CMT de type 4B. $\diamond$

More myelin is not necessarily good

NOUVELLE

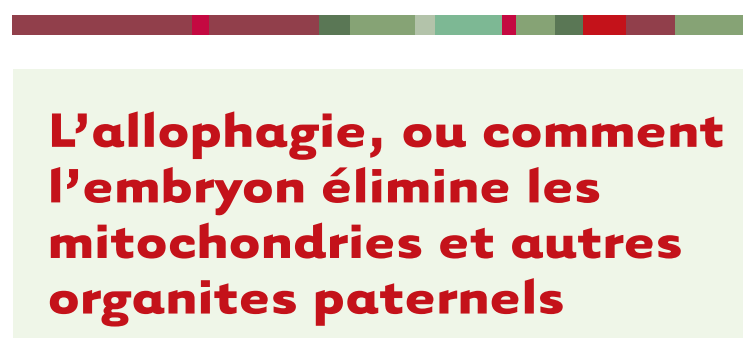

Sara Al Rawi ${ }^{1,2}$, Vincent Galy ${ }^{1,2}$

\section{REMERCIEMENTS}

Nous remercions J.L. Pasquier pour son aide à la réalisation des figures.

\section{CONFLIT D'INTÉRÊTS}

Les auteurs déclarent n'avoir aucun conflit d'intérêts concernant les données publiées dans cet article.

\section{RÉFÉRENCES}

1. Vallat JM, Funalot B. La maladie de Charcot-MarieTooth. Med Sci (Paris) $2010 ; 26: 842-7$.

2. Nave KA, SalzerJL. Axonal regulation of myelination by neuregulin 1. Curr Opin Neurobiol $2006 ; 16: 492-500$.

3. Lee KF, Simon $\mathrm{H}$, Chen $\mathrm{H}$, et al. Requirement for neuregulin receptor erbB2 in neural and cardiac development Nature 1995 ; 378 : 394-8.

4. Riethmacher D, Sonnenberg-Riethmacher $\varepsilon$, Brinkmann V, et al. Severe neuropathies in mice with targeted mutations in the $\varepsilon$ rbB3 receptor. Nature 1997 ; $389:$ : 725-30.

5. Taveggia C, Zanazzi G, Petrylak A, et al. Neuregulin-1 type III determines the ensheathment fate of axons. Neuron $2005 ; 47: 681-94$.

6. Ozçelik M, Coter L, Jacob C, et al. Palsl is a major regulator of the epithelial-like polarization and the extension of the myelin sheath in peripheral nerves. J Neurosci $2010 ; 30: 4120-31$.

7. Cotter L, Ozçelik M, Jacob C, et al. DIg1-PTEN interaction regulates myelin thickness to prevent damaging peripheral nerf overmyelination. Science $2010 ; 328: 1415-8$.

8. Salemena L, Carracedo A, Pandolfi PP. Tenets of PTEN tumor suppression. Cell 2008 ; 133 : 403-14.

9. Contreras-Paredes A, De la Cruz-Hernández $\varepsilon$, Martínez-Ramírez I, et al. $\varepsilon 6$ variants of human papillomavirus 18 differentially modulate the protein kinase B/phosphatidylinositol 3-kinase (akt/PI3K) signaling pathway. Virology $2009 ; 383$ : 78-85.

10. Suter U, Scherer SS. Disease mechanisms in inherited neuropathies. Nat Rev Neurosci 2003 ; 4 : 714-26.
${ }^{1}$ UPMC Université Paris 06, UMR 7622, Laboratoire de biologie du développement, 9, quai Saint-Bernard, F-75005 Paris, France. ${ }^{2}$ CNRS, UMR 7622, Laboratoire de biologie du développement, 9, quai Saint-Bernard, F-75005 Paris, France. vincent.galy@snv.jussieu.fr

se posent alors : quels sont les enjeux liés à l'élimination de l'ADNmt paternel par l'ovocyte? Quelles seraient les conséquences de son maintien pour l'organisme? Comment cette élimination ciblée a-t-elle lieu ? Les mécanismes permettant cette dégradation étaient débattus jusqu'à récemment et plusieurs hypothèses étaient proposées. 